\title{
Table S13
}

\begin{tabular}{|c|c|}
\hline Species & Accession no. \\
\hline Arthrobacter sp. FB24 & CP000454 \\
\hline Bifidobacterium longum $\mathrm{NCC} 2705$ & AE014295 \\
\hline Clavibacter michiganensis subsp. michiganensis NCPPB 382 & AM711867 \\
\hline Corynebacterium diphtheriae NCTC 13129 & BX248353 \\
\hline Corynebacterium glutamicum ATCC 13032 & BX927147 \\
\hline Frankia sp. CcI3 & СР000249 \\
\hline Leifsonia xyli subsp. xyli str. CТCВ07 & AE016822 \\
\hline Mycobacterium smegmatis str. MC2 155 & СР000480 \\
\hline Mycobacterium tuberculosis $\mathrm{H} 37 \mathrm{Rv}$ & AL123456 \\
\hline Nocardia farcinica IFM 10152 & AP006618 \\
\hline Propionibacterium acnes KPA171202 & AE017283 \\
\hline Rhodococcus equi $103 \mathrm{~S}$ & FN563149 \\
\hline Rhodococcus erythropolis PR4 & AP008957 \\
\hline Rhodococcus jostii RHA1 & СР000431 \\
\hline Rhodococcus opacus B4 & AP011115 \\
\hline Rubrobacter xylanophilus DSM 9941 & СР000386 \\
\hline Saccharopolyspora erythraea NRRL2338 & AM420293 \\
\hline Salinispora tropica CNB-440 & СР000667 \\
\hline Streptomyces coelicolor A3(2) & AL645882 \\
\hline Thermobifida fusca YX & СР000088 \\
\hline Tropheryma whipplei TW08/27 & BX072543 \\
\hline
\end{tabular}

